AIRWAY BIOLOGY

\title{
Attenuation of bleomycin induced pulmonary fibrosis in mice using the heme oxygenase inhibitor Zn- deuteroporphyrin IX-2,4-bisethylene glycol
}

\author{
L Atzori, F Chua, S E Dunsmore, D Willis, M Barbarisi, R J McAnulty, G J Laurent
}

Thorax 2004;59:217-223. doi: 10.1136/thx.2003.008979

See end of article for authors' affiliations

Correspondence to: Dr L Atzori, Centre for Respiratory Research, Royal Free and University College Medical School, 5 University Street, London WC1E 6JJ, UK; latzori@ unica.it

Received 17 April 2003 Accepted

25 November 2003

\begin{abstract}
Background: Pulmonary fibrosis is associated with a poor prognosis. The pathogenesis of fibrotic lung disorders remains unclear, but the extent of tissue damage due to the persistent presence of oxidants or proteases is believed to be important. The heme degrading enzyme heme oxygenase $(\mathrm{HO})$ has been found to be expressed in experimental fibrosis, and generation of free iron and carbon monoxide (CO) by $\mathrm{HO}$ has been implicated in oxidant induced lung damage. A study was undertaken to examine the effects of the HO inhibitor Zn-deuteroporphyrin-IX-2,4-bisethylene glycol (Zndtp) on the development of pulmonary fibrosis in the bleomycin model of lung injury and repair.

Methods: Zndtp ( $10 \mu \mathrm{mol} / \mathrm{kg})$ was administered subcutaneously twice daily to mice 1 week following the intratracheal instillation of $0.025 \mathrm{U}$ bleomycin. Animals were killed 10 or 21 days after bleomycin instillation and indices of lung damage and fibrosis were evaluated.

Results: Bleomycin treatment induced pulmonary cytotoxicity, increased levels of active transforming growth factor $\beta$ (TGF- $\beta$ ), enhanced lung collagen accumulation, and decreased glutathione content. Zndtp administration significantly attenuated these indices.

Conclusions: Administration of Zndtp in the bleomycin model resulted in appreciable alveolar cytoprotection and amelioration of pulmonary fibrosis. This molecule and its analogues may warrant further consideration in the treatment of acute lung injury and fibrotic lung disorders.
\end{abstract}

P ulmonary fibrosis is the pathological hallmark of a heterogeneous group of disorders in which excessive collagen accumulation results from a variety of insults to the lung. The clinical prognosis for patients with this condition remains poor, ${ }^{1}$ and limited information on the pathogenetic mechanisms of pulmonary fibrosis has hampered development of new therapeutic interventions. One theory for the development of pulmonary fibrosis centres on the hypothesis that, in response to injury, the damaging effects of oxidants on the alveolar architecture eventually lead to fibroblast activation and collagen production. ${ }^{2}$ This hypothesis is supported by evidence that patients with pulmonary fibrosis have an imbalance between oxidant and antioxidant defence mechanisms in the lung microenvironment. ${ }^{34}$

Heme oxygenase $(\mathrm{HO})$ is the rate limiting enzyme that catalyses the initial step of heme degradation, producing biliverdin and releasing free iron and carbon monoxide (CO). Three isozymes of HO have been identified to date. Whereas HO-2 and HO-3 are constitutively expressed, HO- 1 is upregulated during oxidative stress and has been proposed to have a role in the regulation of inflammatory processes ${ }^{56}$ by acting as a cytoprotective molecule. ${ }^{7}$ Increased expression of HO- 1 has been associated with a number of pulmonary disorders including asthma, ${ }^{8}$ chronic obstructive pulmonary disease, ${ }^{9}$ and lung injury secondary to hypoxia, ${ }^{10}$ hyperoxia, ${ }^{11}$ or bleomycin. ${ }^{12}$ It is also known that HO-l activity, if unchecked, can exert damaging effects as a result of the excessive production of its products-namely, bilirubin, CO, and iron $^{13}$-and the apparent protective role of high levels of $\mathrm{HO}$, particularly in hyperoxia related oxidative stress, has recently been questioned. ${ }^{14-16}$ Likewise, the significance of increased HO expression and function in pulmonary fibrosis remains uncertain.
Parenteral administration of bleomycin, a chemotherapeutic agent, consistently induces an inflammatory alveolitis and alveolar repair that leads to fibrotic extracellular matrix remodelling. ${ }^{17}$ This model has been widely used to study the pathogenetic mechanisms of pulmonary fibrosis in a number of animal species. The principal aim of this study was to evaluate the importance of $\mathrm{HO}$ in the pathogenesis of bleomycin induced lung injury and fibrosis by inhibiting its activity in vivo with the metalloporphyrin Zn-deuteroporphyrin IX-2,4-bisethylene glycol (Zndtp).

\section{METHODS}

\section{Animals}

Experiments were performed using male C57/BL6 mice aged 6-8 weeks. All procedures were approved by the UK Home Office and carried out in accordance with the Animals (Scientific Procedures) Act 1986. Mice were housed under conventional conditions for a week before treatment.

\section{Experimental protocol}

Bleomycin sulphate (BLM, Bleo-Kyowa; Kyowa Hakko Ltd, Slough, UK) was administered as a single intratracheal dose of $0.025 \mathrm{U} / 50 \mu \mathrm{l}$ saline/animal following halothane induced anaesthesia. Control animals received saline alone. Zndtp $(10 \mu \mathrm{mol} / \mathrm{kg})$ was prepared fresh in phosphate buffered saline (PBS, pH 7.4) and administered subcutaneously ( $50 \mu \mathrm{l}$ twice daily) beginning 7 days after bleomycin instillation. Animals were divided into four experimental groups:

Abbreviations: $\mathrm{HO}$, heme oxygenase; LDH, lactate dehydrogenase; MAL, malondialdehyde; TGF- $\beta$, transforming growth factor $\beta$; Zndtp, Zn-deuteroporphyrin IX-2,4-bisethylene glycol 
(1) saline+vehicle, (2) BLM+vehicle, (3) saline+Zndtp, and (4) BLM+Zndtp.

In order to study the effects of Zndtp on alveolar injury and repair preceding fibrotic development, animals were killed 10 days after bleomycin instillation (that is, after 3 days of Zndtp exposure). To evaluate the morphological and biochemical indices of bleomycin induced pulmonary fibrosis, animals were killed 21 days after its instillation ( 14 days after initiation of Zndtp administration).

Groups of 4-11 animals were killed for biochemical and histological analyses on days 10 and 21 by a lethal injection of intraperitoneal pentobarbital followed by aortic exsanguination.

\section{Collagen measurement}

Total lung collagen was determined by measuring the hydroxyproline content using reverse phase high performance liquid chromatography (HPLC) as previously described. ${ }^{18}$ Briefly, powdered lung tissues were weighed and hydrolysed in $6 \mathrm{~N}$ hydrochloric acid at $110^{\circ} \mathrm{C}$ for 16 hours. Hydrolysates were mixed with activated charcoal, filtered through a $0.65 \mu \mathrm{m}$ mesh filter (Millipore, Watford, UK), and an aliquot was removed and dried under vacuum. Hydroxyproline was isolated chromatographically after pre-column derivatisation with 7-chloro-4-nitrobenz-oxo-1,3-diazole (Sigma, Poole, UK). The lung collagen content was calculated based on its hydroxyproline constitution ( $12.2 \%$ by weight) and the results were expressed as mg collagen per lung. ${ }^{18}$

\section{Histopathology}

Lungs were fixed by intratracheal instillation of $4 \%$ paraformaldehyde in PBS at a constant pressure of $25 \mathrm{~cm} \mathrm{H}_{2} \mathrm{O}$. The trachea was ligated and the lungs were excised en bloc and immersed in the same fixative. Following overnight sucrose immersion the lungs were dehydrated through a serial alcohol gradient and embedded in paraffin wax. Sections $(5 \mu \mathrm{m})$ were mounted on glass slides, dewaxed in xylene, and rehydrated before staining with either haematoxylin-eosin or Masson's trichrome. Changes in the amount of extracellular matrix were assessed semi-quantitatively using an Olympus BX40 microscope and Zeiss KS300 Imaging Systems Software (Carl Zeiss Vision GmbH, Germany). Briefly, 20 random fields ( $\times 200$ magnification) were examined per section and five sections were assessed from each lung. Care was taken to match background light and colour intensity and to exclude excessively hypercellular areas. Collagenous matrix was identified by its distinct characteristics on Masson's staining.

\section{Bronchoalveolar lavage}

Bronchoalveolar lavage (BAL) was performed 10 days after bleomycin instillation. The trachea was cannulated and the distal lungs lavaged with six sequential aliquots of $400 \mu$ lice cold sterile saline. A small ( $20 \mu \mathrm{l})$ aliquot of each sample was removed for leucocyte counts and the remaining BAL fluid was centrifuged for 10 minutes at $1500 \mathrm{rpm}\left(4^{\circ} \mathrm{C}\right)$. The total leucocyte count was used as one of several indices of bronchoalveolar inflammation during the acute phase of bleomycin induced damage. Cell free supernatants were kept at $-70^{\circ} \mathrm{C}$ until used.

\section{Biochemical assays}

BAL fluid was analysed for lactate dehydrogenase (LDH) content (CytoTox96 Non-Radioactive Cytotoxicity Assay; Promega, Madison, WI, USA), active transforming growth factor $\beta$ (TGF- $\beta$ ) levels, ${ }^{19}$ malondialdehyde (MDA, Lipid Peroxidation Colorimetric Assay; Oxford Biomedical Research, MI, USA), and bilirubin levels (Total Bilirubin Kit 552-A; Sigma Diagnostic, Poole, Dorset, UK). Measurement of total glutathione in lung tissue homogenates was performed as previously described..$^{20}$

\section{Statistical analysis}

Instat software (GraphPad Software, San Diego, CA, USA) was used to analyse the data. A two tailed Mann-WhitneyWilcoxon test was used for non-parametric comparisons while analysis of variance (ANOVA) with post hoc analysis using the Tukey-Kramer test was used for parametric data. The results are presented as mean (SE) values. A p value of $<0.05$ was considered statistically significant. For primary findings, $95 \%$ confidence intervals (95\% CI) are also included. Confidence intervals for the mean differences between groups are shown for the primary findings.

\section{RESULTS}

Bleomycin instillation resulted in a greater loss in overall body weight than saline treatment, an effect that was not modulated by exposure to Zndtp. However, final mean body weights were not statistically different between bleomycin and saline instillation at the end of either the 10 or 21 day experiments. Bleomycin treatment was also associated with a higher mortality and non-significant increases in lung weight compared with saline treated controls. Zndtp exposure did not alter these parameters appreciably.

\section{Assessment of fibrosis}

\section{Lung collagen content}

Lung hydroxyproline content was determined as a biochemical index of parenchymal collagen accumulation. Figure 1 shows that intratracheal instillation of bleomycin induced a twofold increase in total lung collagen compared with saline treatment at 21 days $(2.10(0.26) \vee v 1.12(0.12) \mathrm{mg} / \mathrm{lung}$, $\mathrm{p}<0.001 ; 95 \%$ CI 1.65 to -0.34 ). Administration of Zndtp to bleomycin treated mice attenuated this increase by approximately $65 \%(1.45(0.20) \vee 2.10(0.26) \mathrm{mg} / \mathrm{lung}, \mathrm{p}<0.05 ; 95 \%$ CI 1.11 to 0.25 ), although levels remained higher than those in saline treated control mice. Zndtp had no effect on basal collagen levels in saline treated animals.

\section{Histopathology}

The extent of pulmonary fibrosis was also assessed histologically. 21 days after saline instillation, lungs of mice receiving either subcutaneous vehicle or Zndtp had a normal architecture (figs 2A and 2C). In contrast, lungs from animals

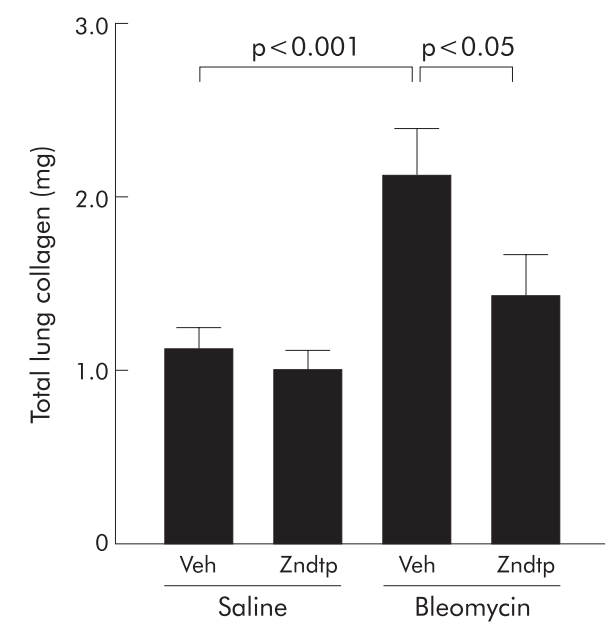

Figure 1 Lung collagen content measured by reverse phase high performance liquid chromatography 21 days after bleomycin or saline instillation ( $n=11$ animals in each treatment group). Veh=vehicle; Zndtp $=$ Zn-deuteroporphyrin IX-2,4-bisethylene glycol. 
treated with bleomycin showed a marked increase in the number of interstitial cells, some with a fibroblastic phenotype, as well as considerable increases in the amount of collagenous extracellular matrix (fig 2B). Zndtp attenuated the morphological changes induced by bleomycin as lungs of mice treated with both bleomycin and Zndtp had milder interstitial changes with fewer fibrotic areas (fig 2D). This observation was validated by semiquantitative morphometric analysis ((BLM+veh) $v$ (saline+veh): $234(28) \% v 100(6) \%$, $\mathrm{p}<0.005$; (BLM+veh) $v($ BLM+Zndtp): 234 (28)\% v 130 (8)\%, $\mathrm{p}<0.05$; fig $2 \mathrm{E})$. Visual inspection did not reveal any gross differences in cellular inflammation between either of the bleomycin treated groups.

\section{BAL fluid analysis}

Leucocyte numbers

Leucocytosis was prominent in BAL fluid 10 days after bleomycin instillation (fig 3). Total leucocyte numbers in bleomycin treated mice were higher than in corresponding saline treated controls ((BLM+veh) $v$ (saline+veh): $3.46(0.19) \vee 0.44(0.31) \times 10^{6} / \mathrm{ml}$ BAL fluid, $\left.\mathrm{p}<0.001\right)$. The administration of Zndtp failed to prevent this leucocytosis $(($ BLM+Zndtp) $v$ (saline+Zndtp): $3.99(0.26) \quad v 0.37$ $(0.03) \times 10^{6} / \mathrm{ml}$ BAL fluid, $\left.\mathrm{p}<0.001\right)$. Percentages of neutrophils, macrophages, and lymphocytes did not differ significantly between the different treatment groups (data not shown).
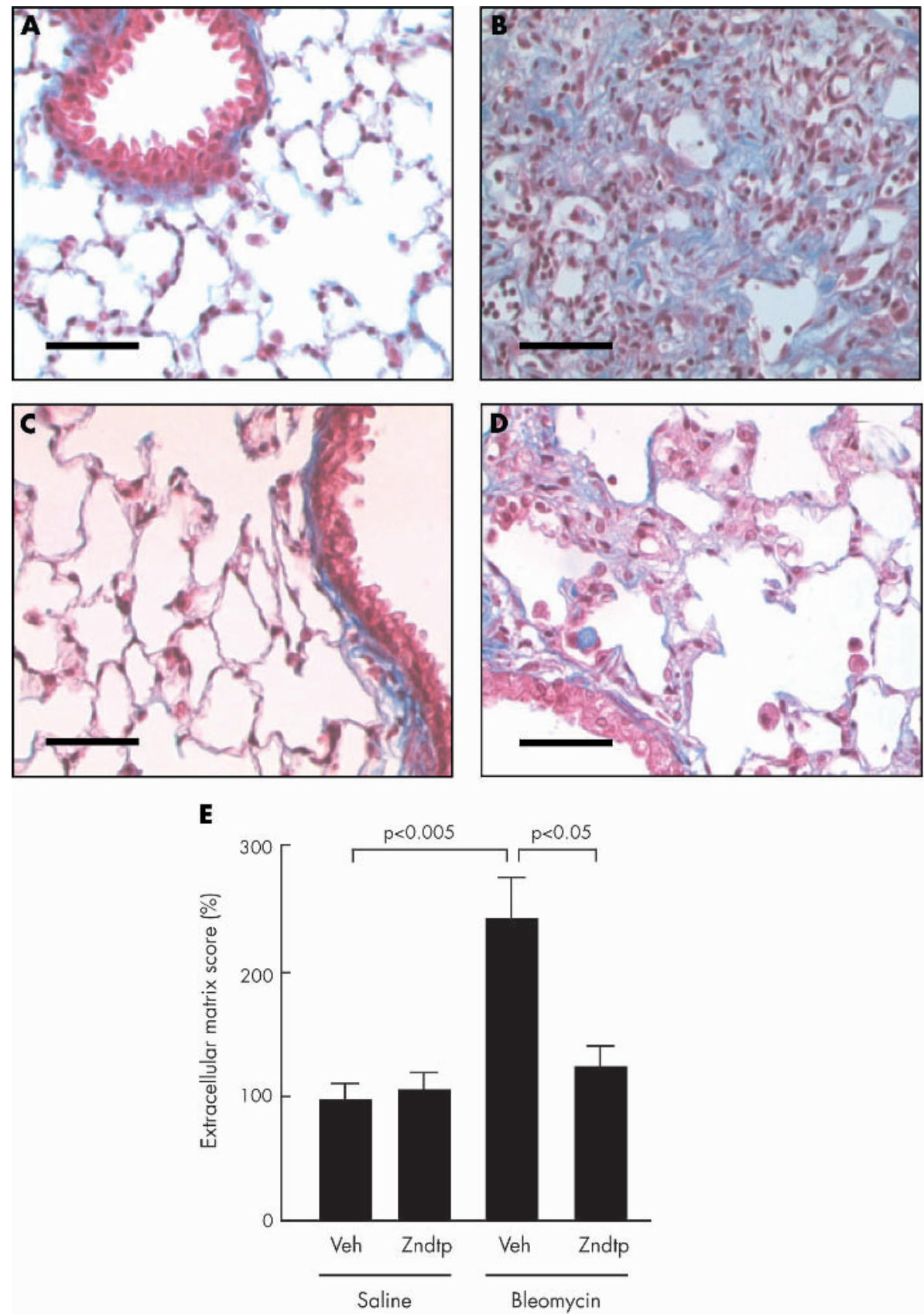

Figure 2 Representative photomicrographs of lung histology. (A) saline+veh, (B) bleomycin+veh, (C) saline+Zndtp, and (D) bleomycin+Zndtp 21 days after bleomycin or saline instillation. Sections were stained with Masson's trichrome. (E) Semiquantitative score of lung extracellular matrix content ( $\mathrm{n}=4$ animals in each treatment group). Bar, $50 \mu \mathrm{m}$. Veh=vehicle; Zndtp =Zn-deuteroporphyrin IX-2,4-bisethylene glycol. 


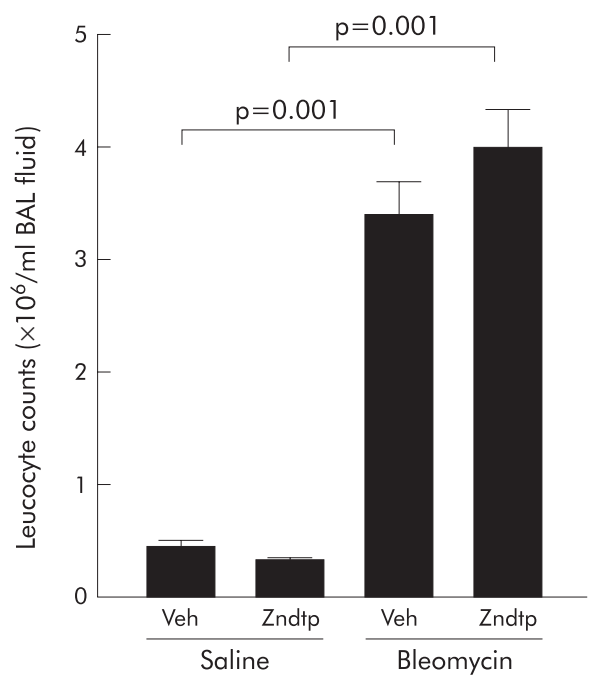

Figure 3 Total number of leucocytes in bronchoalveolar lavage (BAL) fluid preparations collected 10 days after the instillation of bleomycin or saline instillation ( $n=4-8$ animals in each treatment group).

Veh=vehicle; Zndtp=Zn-deuteroporphyrin IX-2,4-bisethylene glycol.

Total bilirubin

Analysis of bilirubin, a secondary product of HO mediated heme degradation, provides an indirect index for assessing HO enzyme activity. Total bilirubin in BAL fluid was measured to confirm the inhibitory effect of Zndtp on HO activity in our model. Figure 4 shows that, in the absence of Zndtp, bleomycin instillation increased the total BAL fluid bilirubin content by twofold compared with saline treated control animals (158.4 (17.9) v 66.0 (9.1) $\mu \mathrm{g} / \mathrm{ml}$ BAL fluid, $\mathrm{p}<0.001)$. Animals in the BLM+Zndtp group had significantly lower bilirubin levels than those in the BLM+veh group ((100.7 (5.6) v 158.4 (17.9) $\mu \mathrm{g} / \mathrm{ml}$ BAL fluid, $\mathrm{p}<0.05)$, although these levels remained higher than those in either of the saline treated groups.

\section{Lactate dehydrogenase (LDH)}

Bleomycin instillation induced a significant increase in the levels of $\mathrm{LDH}$ recovered in BAL fluid $(\mathrm{p}<0.01$, fig $5 \mathrm{~A})$.

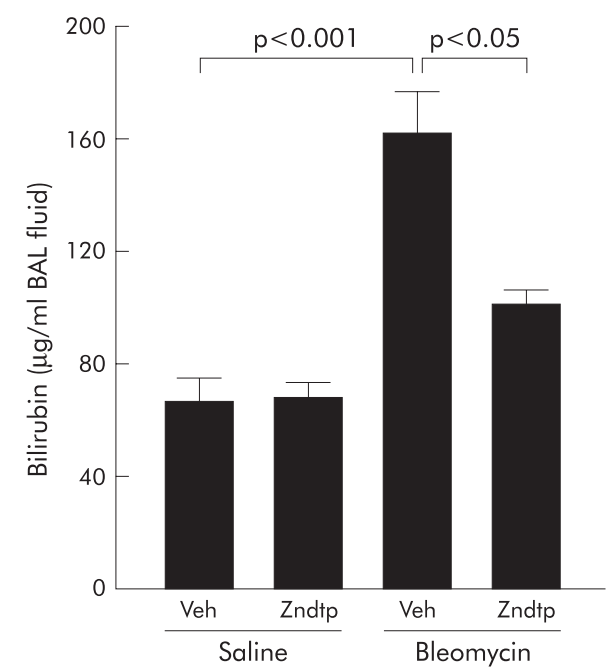

Figure 4 Concentration of total bilirubin in bronchoalveolar lavage (BAL) fluid 10 days after bleomycin instillation $(n=4-8$ animals in each treatment group). Veh= vehicle; Zndtp =Zn-deuteroporphyrin IX-2,4bisethylene glycol.
Administration of Zndtp reduced LDH in bleomycin treated animals ((BLM+Zndtp) $v$ (BLM+veh): 0.048 (0.015) $v 0.293$ $(0.098)$ relative absorbance units, $p<0.01)$ to levels comparable with saline treated controls. Zndtp also appeared to have a small effect on LDH levels in saline treated animals.

\section{Malondialdehyde (MDA)}

Malondialdehyde (MDA), a byproduct of the oxidation of polyunsaturated fatty acids, was measured as an index of oxidative stress induced lipid peroxidation. Figure 5B shows that levels of MDA were almost three times higher following bleomycin treatment than following saline instillation (288 (34)\% v $\quad 100 \quad(21) \%$ from basal values, $\mathrm{p}<0.01)$. Administration of Zndtp significantly abrogated this increase $((\mathrm{BLM}+\mathrm{Zndtp}) \quad v \quad(\mathrm{BLM}+\mathrm{veh}): \quad 178 \quad(25) \% \quad v \quad 288 \quad(34) \%$, $\mathrm{p}<0.04)$. MDA levels in the BLM+Zndtp group were not significantly different from those in either of the saline treated control groups.
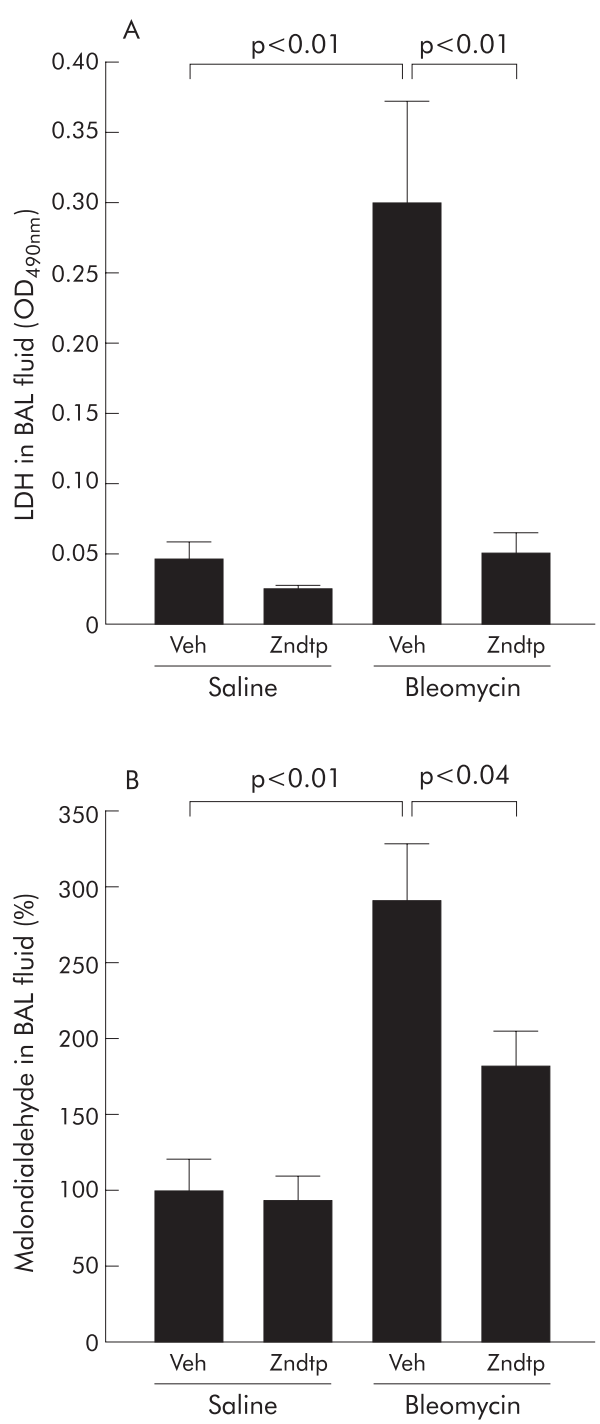

Figure 5 (A) Extracellular abundance of lactate dehydrogenase (LDH) and (B) malondialdehyde (MDA) concentration in the bronchoalveolar lavage (BAL) fluid 10 days after bleomycin instillation ( $n=4-8$ animals in each treatment group). Veh = vehicle; $Z n d t p=Z n$-deuteroporphyrin IX2,4-bisethylene glycol. 


\section{Active TGF- $\beta$}

Figure 6 shows that the highest levels of active TGF- $\beta$ in BAL fluid were measured in the BLM+veh group (0.35 (0.028) ng/ $\mathrm{ml}$ BAL fluid). Replacement of vehicle with Zndtp in bleomycin treated mice significantly reduced this rise in TGF- $\beta(0.28(0.02) \mathrm{ng} / \mathrm{ml}$ BAL fluid, $\mathrm{p}<0.05)$. Levels of active TGF- $\beta$ in the saline treated controls receiving either vehicle or Zndtp did not differ from each other.

\section{Lung tissue glutathione}

Glutathione was measured as an indicator of the pulmonary antioxidant index. Figure 7 shows that, in the absence of Zndtp, the levels of total glutathione were halved in lung tissue from bleomycin treated mice compared with saline treated controls ((BLM+veh) $v$ (saline+veh): 1.35 (0.28) $v$ $2.93(0.29) \mu \mathrm{g} / \mathrm{mg}$ lung, $\mathrm{p}<0.05$; 95\% CI 0.226 to 2.954$)$. Administration of Zndtp preserved total glutathione levels and prevented their reduction $((\mathrm{BLM}+\mathrm{Zndtp}) v(\mathrm{BLM}+\mathrm{veh})$ : $2.52(0.20) v 1.35(0.28) \mu \mathrm{g} / \mathrm{mg}$ lung, $\mathrm{p}<0.05 ; 95 \%$ CII.175 to $1.643)$.

\section{DISCUSSION}

This study was undertaken to examine the potential role of $\mathrm{HO}$ in oxidative lung injury resulting from the administration of bleomycin. Using techniques of global gene analysis, Kaminski et al have previously shown that $\mathrm{HO}$ expression is strongly induced 14 days after the initiation of bleomycin induced lung injury. ${ }^{12}$ However, the pathogenetic role of $\mathrm{HO}$ in lung injury was not addressed. We therefore chose to start inhibition of HO 7 days after the intratracheal instillation of bleomycin. Our data indicate that administration of the metalloporphyrin Zndtp, a potent inhibitor of HO activity, significantly attenuates bleomycin induced lung damage and pulmonary fibrosis, as assessed biochemically and histologically. These data are consistent with the hypothesis that HO has a role in the pathways that regulate lung repair.

\section{Inhibition of bleomycin induced lung injury and fibrosis by Zndtp \\ Lung injury}

The pathophysiology of bleomycin induced lung injury typically consists of two overlapping stages: an early inflammatory phase characterised by leucocyte infiltration and injury to alveolar epithelial cells, and a subsequent

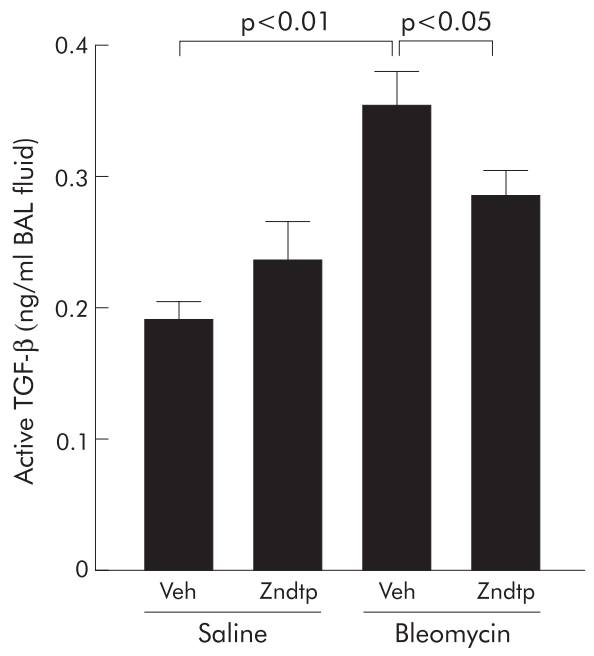

Figure 6 Levels of active transforming growth factor $\beta$ (TGF- $\beta$ ) in bronchoalveolar lavage (BAL) fluid 10 days after the intratracheal instillation of bleomycin ( $n=4-8$ animals in each treatment group). Veh =vehicle; Zndtp =Zn-deuteroporphyrin IX-2,4-bisethylene glycol. fibroproliferative phase with matrix remodelling and fibrosis. Recent evidence suggests that aberrant epithelial-mesenchymal cell interactions might play a central role in the pathogenesis of fibrotic lung disease. ${ }^{21}$ Damage to the alveolar epithelium may be induced by reactive oxygen species, proteases, or inflammatory mediators, resulting in aberrant tissue repair that culminates in fibrosis.

Zndtp appears to minimise alveolar cytotoxicity, as evidenced by decreased lipid peroxidation products and reduced levels of extracellular LDH. Despite these biochemical changes, Zndtp did not affect leucocyte accumulation in the alveolar space. This observation suggests that Zndtp can have effects independent of inhibition of inflammation and that inflammatory mechanisms may not play a dominant role in the pathological events preceding fibrotic development. Such a notion has been the subject of recent debate, particularly in relation to the pathogenesis of human pulmonary fibrosis. ${ }^{22}$

\section{Lung fibrosis}

These studies provide the first evidence that the HO inhibitor Zndtp can significantly reduce lung collagen accumulation induced by the fibrogenic agent bleomycin. This protective effect was achieved without affecting basal collagen levels and was accompanied by a parallel decrease in the extracellular matrix score assessed by semiquantitative image analysis of stained histological sections. Although small areas of interstitial thickening and mild fibrosis were present in lungs from Zndtp treated mice exposed to bleomycin, these abnormalities were minor compared with changes in the lungs of mice receiving bleomycin alone. In the latter, large portions of the lung parenchyma were almost completely obliterated by dense fibrotic tissue similar to lesions seen in patients with idiopathic pulmonary fibrosis.

\section{Potential mechanisms of protection by Zndtp}

A number of metalloporphyrins can both inhibit $\mathrm{HO}-1$ activity and regulate $\mathrm{HO}-1$ gene expression at the transcription level. ${ }^{23}$ Zndtp is regarded as the most potent inhibitor of $\mathrm{HO}$ activity. ${ }^{24}$ Evidence of HO inhibition in the current study was demonstrated by the finding of reduced bilirubin in the alveolar space of Zndtp treated mice receiving bleomycin. Interestingly, Zndtp had no effect on bilirubin levels in saline treated animals. One possible explanation may be that Zndtp

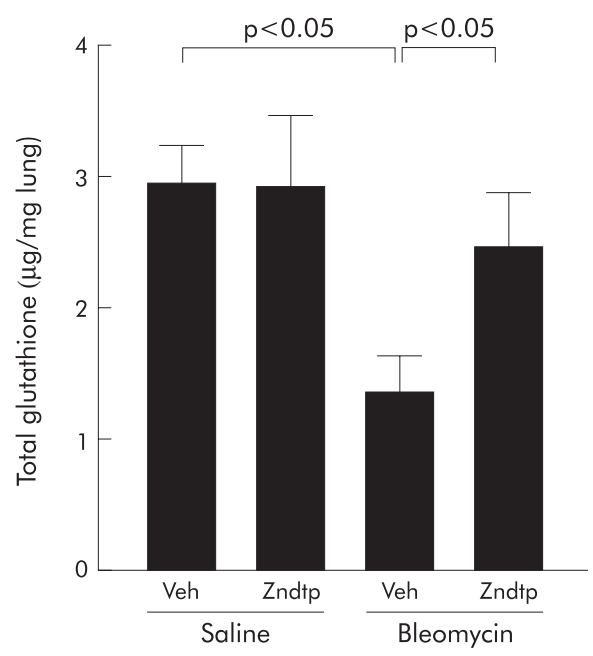

Figure 7 Levels of total glutathione in bronchoalveolar lavage (BAL) fluid 21 days after bleomycin instillation ( $n=4-8$ animals in each treatment group). Veh= vehicle; Zndtp $=Z n$-deuteroporphyrin IX-2,4bisethylene glycol. 
selectively targets inducible $\mathrm{HO}-1$, levels of which rise as a result of lung injury.

There are at least two possible explanations as to why Zndtp might protect against the detrimental effects of bleomycin on the lung. Firstly, inhibition of HO suppresses the generation of potentially cytotoxic heme derived end products. One of these (ferrous iron) is an essential co-factor in bleomycin induced DNA cleavage and can also promote the pro-oxidant effects of HO- $1 .{ }^{15}$ Secondly, the antioxidant properties that are intrinsic to the metalloporphyrin itself may act to restore alveolar antioxidant integrity, thus promoting epithelial cell repair and limiting ongoing injury to this critical barrier. These two potential mechanisms are discussed further below.

\section{Reduced generation of heme derived products}

Levels of tissue HO increase in response to oxidative injury. This increase is thought to protect against hyperoxic cellular injury in vitro ${ }^{12}$ and in vivo. ${ }^{25}$ In addition, HO mediated degradation of heme prevents the native substrate from exerting its own cytotoxic effects. ${ }^{7}$ It is becoming increasingly apparent that the role of HO- 1 in tissue injury is complex and is determined by its local concentration and temporal prominence. ${ }^{815}$ The products of heme catabolism may be harmful, as suggested by studies in which targeted overexpression of HO-1 in alveolar type II cells has been shown to increase the severity of hyperoxic lung injury. ${ }^{26}$ Transpulmonary delivery and overexpression of the HO-l gene in vivo has also been associated with increased tissue oxidative burden and lung injury. ${ }^{14}$

Tsuburai and colleagues have reported that adenovirus mediated transfection of HO-l can attenuate the intensity of bleomycin induced pulmonary fibrosis. ${ }^{27}$ However, their study differs from ours in that unequivocal HO-l protein expression was induced before bleomycin treatment, with levels peaking approximately 1 week after the instillation of bleomycin. It is interesting to speculate that such early induction of HO-l might selectively counteract the acute injurious effects of bleomycin, thereby impeding the development of subsequent fibroproliferation. It is likely that differences in the tissue concentration and temporal prominence of HO-l are important to the different pathological outcome in our study and that of Tsuburai et al. ${ }^{27}$ In our study Zndtp was given only after the initial inflammatory phase. This may account for the reduced fibrosis in both overexpression studies and our inhibitory study, suggesting that $\mathrm{HO}$ is cytoprotective in the early inflammatory phase and profibrotic in the later fibroproliferative phase of bleomycin induced lung toxicity. Furthermore, early expression of HO-1 in the study by Tsuburai and colleagues did not protect against pulmonary fibrosis induced by Fas-Fas ligand crosslinking, arguing for the existence of different molecular pathways governing tissue fibrosis. ${ }^{27}$

\section{Direct antioxidant effects}

Bleomycin concomitantly binds metal ions and DNA, generating oxygen derived radicals that can directly cleave macromolecules, including DNA. Ferrous iron, generated by HO- 1 mediated catabolism of heme, is a central co-factor in bleomycin induced DNA damage. Bleomycin also promotes the depletion of endogenous antioxidant defences, exacerbating oxidant mediated tissue injury. Consequently, strategies aimed at reducing oxidative stress have been successful in decreasing bleomycin induced lung injury and fibrosis induced by this agent. ${ }^{28}$ In our study the ability of Zndtp to ameliorate lipid peroxidation and prevent depletion of lung glutathione stores suggests that its antifibrotic activity in the bleomycin model is mediated in part by its antioxidant properties.
It is now recognised that the effects of $\mathrm{HO}-1$, both protective and deleterious, are likely to be cell specific. Alveolar type II cells have more robust antioxidant defences, partly by having increased glutathione reserves. Paradoxically, the higher concentrations of HO-l in these cells may predispose them to HO-1 mediated injury. A number of in vivo studies have reported findings consistent with this line of thinking. ${ }^{14}{ }^{26}$ Our findings of reduced cellular injury and enhanced antioxidant protection further suggest that the primary recipient of Zndtp mediated cytoprotection may be the alveolar type II cell. Metalloporphyrins have themselves been shown to ameliorate alveolar type II damage in bleomycin induced lung injury. ${ }^{29}$

Finally, our contention that the cytoprotection afforded by HO- 1 inhibition most probably results from a combination of diminished generation of toxic heme metabolites and increased tissue antioxidant capacity is consistent with findings in HO- 1 deficient mice ${ }^{16}$ and transfected cells lacking HO- 1 activity. ${ }^{30}$

\section{TGF- $\beta$ as a common final mediator in bleomycin induced pulmonary fibrosis}

TGF- $\beta$ is the most potent fibrogenic mediator characterised to date, with a propensity for enhancing collagen gene expression and protein deposition. ${ }^{31}$ Animal studies have also shown that inhibition of TGF- $\beta$ activity ${ }^{32}$ or its signalling ${ }^{33}$ can abrogate bleomycin induced pulmonary fibrosis. Furthermore, overexpression of endogenous TGF- $\beta$ is a strong stimulus for the induction of pulmonary fibrosis. ${ }^{34}$ Our data show that Zndtp attenuates bleomycin induced increases in active TGF- $\beta$ levels. The pathogenetic link between TGF- $\beta$ and oxidative injury in pulmonary fibrosis is poorly understood. Nonetheless, increased alveolar epithelial sensitivity to oxidative stress, manifested in part by decreased antioxidant protection, is believed to be important. $^{3}$ More recently, the finding that TGF- $\beta$ mediated depletion of alveolar glutathione stores may promote acute lung injury has provided a possible link between TGF- $\beta$ and impaired antioxidant mechanisms in the lung. ${ }^{35}$ In reality, Zndtp most probably imparts its protective effects on tissue repair via different mechanisms, including the regulation of injurious mediator production and modulation of alveolar responses to oxidant stimuli.

In conclusion, this study shows that parenteral delivery of the HO metalloporphyrin inhibitor Zndtp limits the development of bleomycin induced pulmonary fibrosis. Its administration is associated with reduced lung cell damage, pulmonary pathology, collagen accumulation, and TGF- $\beta$ activity. Our data suggest that altering pulmonary oxidant/ antioxidant imbalances by targeting HO-1 may be worthy of further investigation as we explore new approaches to the treatment of fibrotic lung disorders.

\section{ACKNOWLEDGEMENTS}

The authors thank Steve Bottoms for technical assistance. This study was supported by the Wellcome Trust (programme grant 051154 and a short term travel grant awarded to LA).

\section{Authors' affiliations \\ F Chua, M Barbarisi, R J McAnulty, G J Laurent, Centre for Respiratory Research, Royal Free and University College London Medical School, London, UK \\ L Atzori, Department of Toxicology, University of Cagliari, Cagliari, Italy S E Dunsmore, Department of Pulmonary Medicine and Critical Care Medicine, Brigham and Women's Hospital, Boston, USA \\ D Willis, Department of Pharmacology, University College London, London, UK}

LA and FC contributed equally to the work. 


\section{REFERENCES}

1 American Thoracic Society/European Respiratory Society. International multidisciplinary consensus classification of the idiopathic interstitial pneumonias. Am J Respir Crit Care Med 2002; 165:277-304.

2 Cook DN, Brass DM, Schwartz DA. A matrix for new ideas in pulmonary fibrosis. Am J Respir Cell Mol Biol 2002;27:122-4.

3 Cantin AM, Hubbard RC, Crystal RG. Glutathione deficiency in the epithelial lining fluid of the lower respiratory tract in idiopathic pulmonary fibrosis. Am Rev Respir Dis 1989;139:370-2.

4 MacNee W, Rahman I. Oxidants/antioxidants in idiopathic pulmonary fibrosis. Thorax 1995;50:S53-8.

5 Willis D, Moore AR, Frederick R, et al. Heme oxygenase: a novel target for the modulation of the inflammatory response. Nature Med 1996;2:87-90.

6 Morse D, Choi AMK. Heme oxygenase-1. The emerging molecule has arrived. Am J Respir Cell Mol Biol 2002;27:8-16.

7 Otterbein LE, Choi AMK. Heme oxygenase: colors of defense against cellular stress. Am J Physiol (Lung Cell Mol Physiol) 2000;279:L1029-37.

8 Horvath I, Donnelly LE, Kiss A, et al. Raised levels of carbon monoxide are associated with an increased expression of heme oxygenase- 1 in airway macrophages in asthma: a new marker of oxidative stress. Thorax 1998;53:668-72.

9 Yamada N, Yamaya M, Okinaga S, et al. Microsatellite polymorphism in the heme oxygenase-1 gene promoter is associated with susceptibility to emphysema. Am J Hum Genet 2000;66:187-95.

10 Carraway MS, Ghio AJ, Carter JD, et al. Expression of heme oxygenase- 1 in the lung in chronic hypoxia. Am J Physiol Lung Cell Mol Physiol 2000;278:L806-12.

11 Lee PJ, Alam J, Wiegand GW, et al. Overexpression of heme oxygenase-1 in human pulmonary epithelial cells results in cell growth arrest and increased resistance to hyperoxia. Proc Nat Acad Sci USA 1996;93:10393-8.

12 Kaminski N, Allard JD, Pittet JF, et al. Global analysis of gene expression in pulmonary fibrosis reveals distinct programs regulating lung inflammation and fibrosis. Proc Natl Acad Sci USA 2000;97:1778-83.

13 Dong Z, Lavrovski Y, Manjeri A, et al. Heme oxygenase-1 in tissue pathology. The Yin and the Yang. Am J Pathol 2000;156:1485-8.

14 Weng Y-H, Tatarov A, Bartos BP, et al. HO-1 expression in type II pneumocytes after transpulmonary gene delivery. Am J Physiol (Lung Cell Mol Physiol) 2000;278:L1273-9.

15 Suttner DM, Dennery PA. Reversal of HO-1 related cytoprotection with increased expression is due to reactive iron. FASEB J 1999;13:1800-9.

16 Dennery PA, Visner G, Weng Y-H, et al. Resistance to hyperoxia with heme oxygenase-1 disruption: role of iron. Free Radic Biol Med 2003;34:124-33.

17 Thrall RS, Scalise PJ. Bleomycin. In: Pulmonary fibrosis. New York: Marcel Dekker, 1995:230-92

18 Campa JS, McAnulty RJ, Laurent GJ. Application of high-pressure chromatography to studies of collagen production by isolated cells in culture. Anal Biochem 1990;186:257-63.
19 Abe $M$, Harpel JG, Metz CN, et al. An assay for transforming growth factor- $\beta$ using cells transfected with a plasminogen activator-1 promoter-luciferase construct. Anal Biochem 1994;216:276-84.

20 Montaldo C, Cannas E, Ledda M, et al. Bronchoalveolar glutathione and nitrite/nitrate in idiopathic pulmonary fibrosis and sarcoidosis. Sarcoid Vasc Diffuse Lung Dis 2002;19:54-8.

21 Pardo A, Selman M. Idiopathic pulmonary fibrosis: new insights into its pathogenesis. Int J Biochem Cell Biol 2002;34:1534-8.

22 Gauldie J. Inflammatory mechanisms are a minor component of the pathogenesis of idiopathic pulmonary fibrosis. Am J Respir Crit Care Med 2002; 165: 1205-8.

23 Shan Y, Pepe J, Lambrecht RW, et al. Mapping of the chick heme oxygenase1 proximal promoter for responsiveness to metalloporphyrins. Arch Biochem Biophys 2002;399:159-66.

24 Chernick RJ, Martasek P, Levere RD, et al. Sensitivity of human tissue heme oxygenase to a new synthetic metalloporphyrin. Hepatology 1989;10:365-9.

25 Otterbein LE, Kolls JK, Mantell LL, et al. Exogenous administration of heme oxygenase- 1 by gene transfer provides protection against hyperoxia-induced lung injury. J Clin Invest 1999:103:1047-54.

26 Tom DJ, Rogers LK, McNaughton KM, et al. Mice expressing a heme oxygenase-1 (HO-1) transgene driven by a surfactant protein $C$ (SPC) promoter are more susceptible to hyperoxic lung injury (Abstract). Pediatr Res 1999:45:71A.

27 Tsuburai T, Suzuki M, Nagashima Y, et al. Adenovirus-mediated transfer and overexpression of heme oxygenase 1 cDNA in lung prevents bleomycininduced pulmonary fibrosis via a Fas-Fas ligand-independent pathway. Hum Gene Ther 2002;13:1945-60.

28 Tamagawa K, Taooka Y, Maeda A, et al. Inhibitory effects of a lecithinized superoxide dismutase on bleomycin-induced pulmonary fibrosis in mice. Am J Respir Crit Care Med 2000;161:1270-84.

29 Oury TD, Thakker K, Menache M, et al. Attenuation of bleomycin-induced pulmonary fibrosis by a catalytic antioxidant metalloporphyrin. Am J Respir Cell Mol Biol $2001 ; 25: 164-9$.

30 Hori R, Kashiba M, Tomas T, et al. Gene transfection of H25A mutant heme oxygenase-1 protects cells against hydroperoxide-induced cytotoxicity. J Biol Chem 2002;277:10712-8.

31 Marshall RP, McAnulty RJ, Laurent GJ. The pathogenesis of pulmonary fibrosis: is there a fibrosis gene? Int J Biochem Cell Biol 1997;29:107-20.

32 Giri SN, Hyde DM, Hollinger MA. Effect of antibody to transforming growth factor- $\beta$ on bleomycin-induced accumulation of lung collagen in mice. Thorax 1993;48:959-66.

33 Wang Q, Wang Y, Hyde DM, et al. Reduction of bleomycin induced lung fibrosis transforming growth factor- $\beta$ soluble receptor in hamsters. Thorax 1999;54:805-12.

34 Sime PJ, Xing Z, Graham FL, et al. Adenovector-mediated gene transfer of active transforming growth factor-betal induces prolonged severe fibrosis in rat lung. J Clin Invest 1997;100:768-76.

35 Pittet J-F, Griffiths MJD, Geiser T, et al. TGF- $\beta$ is a critical mediator of acute lung injury. J Clin Invest 2001;107:1537-44. 